form polyphenolic substances containing methylene linkages between the different phenolic groups, such as the crystalline dihydroxy-diarylmethanes and the more complicated high molecular phenol-formalde. hyde resins.' The problem of finding conditions, under which anhydro-2- or 4-hydroxy-arylalcohols would be formed, and, in addition, would be sufficiently resistant to the formation of the condensed phenol-formaldehyde products mentioned, at the required high temperature (in excess of $250^{\circ}$ ), could therefore be expected to be rather complicated.

2.6-Dihydroxymethyl-4-methyl-phenol, $\mathrm{HO}-\mathrm{C}_{6} \mathrm{H}_{3}\left(\mathrm{CH}_{2} \mathrm{OH}\right)_{2} \mathrm{CH}_{3}$, was chosen as subject of a detailed investigation. Preliminary experiments showed that, dry distilled, this substance decomposes with the formation of small amounts of 2.4dimethyl-phenol and 2.4.6-trimethyl-phenol, the two theoretically possible methylated phenols, in addition to a considerable amount of $p$-cresol. The formation of $p$-cresol is due to the intermediate formation of phenol-formaldehyde condensation products consisting of two or more aromatic groups connected by methylene linkages, which, as already shown by Megson ${ }^{4}$, and confirmed by us, decompose with formation of the original phenols in addition to traces of methylated phenols (generally less than 2 pər cent). This is completely analogous to the formation of aniline from anhydro-4-amino-benzylalcohol owing to the intermodiate formation of aniline-formaldehyde resins.

Attempts to heat 2.6-dihydroxymethyl-4-methylphenol in presenee of alkaline materials of different strength in the hope of finding conditions which would inhibit the undesired condensation proved to be successful. The amount of methylated phenols varies in presence of different alkaline materials. We have found that alkalis of medium strength such as the hydroxides or oxides of the alkaline earths or the borates of the alkali metals give the best results; yields as high as 25-30 per cent of 2.4.6-trimethylphenol, in addition to some 2.4-dimethyl-phenol, are obtained. The corresponding salts of 2.6-dihydroxymethyl-4-methyl-phenol behave similarly.

The reaction discussed is characteristic for all 2-or 4-hydroxymethyl-phenols and other substances able to form at high temperatures anhydro-2- or 4hydroxymethyl-phenols ; for example, 2- or 4aminomethyl-phenols, $\mathrm{R}_{2} \mathrm{~N}-\mathrm{CH}_{2}-\mathrm{Ar}-\mathrm{OH}^{5}$.

The bearing of these results on the theory of the formation and structure of phenol-formaldehyde resins and the possibility of making the above results the basis of an economic process for the preparation of nuclear methylated phenols, especially 2.4.6-trimethylphenol, 2.3.5-trimethylphenol, 2.3.5.6-tetra. methylphenol, 2.3.4.6-tetramethylphenol, will be discussed in a more detailed paper to be published elsewhere ${ }^{5}$

We thank the Calico Printers' Association, Ltd., Manchester, for permission to publish this letter.

M. G. Barclay.

A. Burawoy.

G. H. Thomson.

College of Technology,

Manchester 1. April 13.

${ }^{1}$ Hofmann, Proc. Roy. Soc., 12, 576 (1863).

${ }^{2}$ cf. Rice and Rice, “The Aliphatic Free Radicals"' (Baltimore, 1935), pp. $91,142$.

$3 \mathrm{cf}$. in the meantime, Burawoy, B. Pat., 539,747 .

- Trans. Far. Soc., 32, 336 (1935).

${ }^{5}$ cf. Furawoy, B. Pat., 545,382.

\section{Theory of Large Elastic Deformations}

When rubber is subjected to a large elastic deformation, which may be assumed to take place without change of volume, it ceases to be isotropic, and the attempt to relate the stresses and strains in different directions may be a matter of some difficulty. However, if the assumption is made that Hooke's law is obeyed in simple shear in any isotropic plane, it is possible, as Mooney has shown ${ }^{1}$, to deduce certain relations which represent the elastic behaviour under the most general type of homogeneous deformation. If such a deformation is defined by the three principal strains, $\lambda_{1}, \lambda_{2}$ and $\lambda_{3}$ (where $\lambda_{i}$ is the ratio of final to initial length along the $i$-strain axis), the expression for the work of deformation $W$ is

$$
W=\frac{G}{4} \sum_{i=1}^{3}\left(\lambda_{i}-\frac{1}{\lambda_{i}}\right)^{2}+\frac{H}{4} \sum_{i=1}^{3}\left(\lambda^{2}-\frac{1}{\lambda_{i}^{2}}\right)
$$

This equation, which rests on an argument of a most general character, leaves two constants $G$ and $H$ to be determined by the specific properties of the material. The constant $G$ may be identified with the ordinary modulus of rigidity, but the physical significance of $H$ is not easy to grasp. The ratio $H / G$ is related to the difference of behaviour under different types of deformation, and is termed by Mooney the "coefficient of asymmetry".

Approaching the subject from a different angle, Wall ${ }^{2}$ has recently derived stress-strain relations for an idealized network of molecules, on the basis of the kinetic theory of elasticity. He finds a linear stress-strain relation in shear, and by comparing his equation for elongation with that given by the more general theory of Mooney (equation 1) he is able to relate the constants $G$ and $H$ to a single molecular parameter. The result is

$$
G=H=N \boldsymbol{k} T,
$$

where $N$ is the effective number of molecules per c.c., $\boldsymbol{k}$ is Boltzmann's constant and $T$ the absolute temperature. Combining this result with equation (1) leads to the simpler form

$$
W=\frac{G}{2}\left(\lambda_{1}{ }^{2}+\lambda_{2}{ }^{2}+\lambda_{3}{ }^{2}-3\right)
$$

for the strain energy in the most general type of homogeneous deformation. It follows, therefore, that the description of rubber-like elasticity does not necessarily require the use of two independent physical constants. Whether in fact the behaviour of a given rubber can be satisfactorily represented in terms of a single physical constant is, of course, a matter for experimental investigation.

Our unpublished experiments show that Wall's relations do in fact represent the behaviour of vulcanized rubber in elongation, compression and shear with reasonable accuracy up to moderately large deformations.

If Wall's theory is assumed, then having once used it to derive the simplified form (2) of Mooney's equation, one may proceed to apply this equation to any problem involving the more complex types of deformation of rubber, without further reference to the molecular theory.

British Rubber Producers'

L. R. G. Treloar.

Research Association, Laboratories, Welwyn Garden City. May 5.

${ }^{1}$ J. Appl. Phys., 11, 582 (1940).

2 J. Chem, Phys., 10, 485 (1942). 